The role of laboratory testing in the development of rotor aerodynamics (review)

\title{
Okulov, Valery
}

Published in:

Thermophysics and Aeromechanics

Link to article, DOI:

$10.1134 /$ S0869864318010018

Publication date:

2018

Document Version

Peer reviewed version

Link back to DTU Orbit

Citation (APA):

Okulov, V. (2018). The role of laboratory testing in the development of rotor aerodynamics (review).

Thermophysics and Aeromechanics, 25(1). https://doi.org/10.1134/S0869864318010018

\section{General rights}

Copyright and moral rights for the publications made accessible in the public portal are retained by the authors and/or other copyright owners and it is a condition of accessing publications that users recognise and abide by the legal requirements associated with these rights.

- Users may download and print one copy of any publication from the public portal for the purpose of private study or research.

- You may not further distribute the material or use it for any profit-making activity or commercial gain

- You may freely distribute the URL identifying the publication in the public portal

If you believe that this document breaches copyright please contact us providing details, and we will remove access to the work immediately and investigate your claim. 
DOI: $10.1134 / \mathrm{S} 0869864318010018$

\title{
The role of laboratory testing in the development of rotor aerodynamics (review)
}

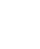

\section{V.L. Okulov}

The Technical Unviersity of Denmark, Lyngby, Denmark

Kutateladze Institute of Thermophysics SB RAS, Novosibirsk, Russia

E-mail: vaok@dtu.dk

(Received July 5, 2017; in revised form September 7, 2017)

\begin{abstract}
The aim of the review is to assess the value of model experimental studies for the development of classical rotor aerodynamics as well as to describe the most significant recent results stimulated by intense development of wind power.
\end{abstract}

Key words: rotor aerodynamics, vortex wake behind the rotor, wind power plant, interaction between the rotor and the wake, power loss.

\section{Introduction}

The current stage of rotor aerodynamics development is undoubtedly associated with the development of wind power, with the transition from single wind turbines to a network of wind power plants (WPP) and their transformation into the industrial sector - the most important component of the global energy potential (Gupta, 2015).

In WPP, as a rule, several industrial wind turbines are combined into a single network the "wind farm" (WF) that is included in the unified energy systems. Currently, the basis for the development of industrial wind energy, including in Russia (Fortov, Popiel, 2014; Gordeev et al., 2012), are major WFs consisting of a large number of wind turbines. Sometimes they consist of up to 100 or more wind turbines. The rate of development of WF in Europe and America is currently comparable to that of nuclear energy development in the second half of the twentieth century, and might have even surpassed it already. Accordingly, the interest of modern scholars in rotor aerodynamics has significantly increased. Today, as in the productive aviation era of Joukowsky and Prandtl and their schools, there has been a new phase of intensive scientific development of rotor aerodynamics (Okulov et al., 2013). It is primarily associated with solving the tasks of efficiency increase of wind turbines, arranged one behind the other and integrated in compact groups of WF. This requires additional optimization of work regimes of turbines that fall in the vortex wake behind the rotor of the preceding WF, where the deviation from the estimated optimal performance is due to a significant reduction of wind velocity inside the wake compared to the main flow. This velocity deficit and

\footnotetext{
* The work was financially supported by the Russian Science Foundation (Grant 14-19-00487).
}

(C) V.L. Okulov, 2018 
additional increase in the level of non-stationary pulsations due to interaction with vortex structures coming off the rotor blades significantly worsen the working conditions of subsequent turbines, falling in the wake in the farm (Vermeer et al., 2003). Average power losses in a large wind farm make up from 10 to $20 \%$ due to interaction with wakes of preceding wind turbines. Therefore, the development of rotor aerodynamics today focuses primarily on the study of the dynamics of vortex rotor wakes and the construction of new aerodynamic models of interaction for the search for optimal configurations of WF (Sørensen et al., 2013). These tasks are associated with the need to examine the far inter-rotor interaction. So far the theory of rotor machines has been mostly considering the problem of near interaction between the stator and rotor or two rotors for two-stage propellers, when the vortex system generated by the blades of the first stage immediately interacts with the blades of the subsequent one.

New requirements to the description of rotor interaction at large distances in WF assume addressing and solving more complex problems of classical aerodynamics related to the study and description of complex swirling flows in the wakes, interactions with helical vortices and their multiplets. It gives a new impetus to the study of fundamental problems in vortex dynamics and the theory of stability of vortex structures (see, e.g., Okulov, Sørensen, 2007; Walther et al., 2007; Felli et al., 2011; Quaranta, Leweke, 2015; Okulov, 2016). The need of the industry in WF stimulated the development of some semi-empirical and engineering methods of calculation of their work (Barthelmie et al., 2009; Larsen et al., 2013; Nygaard, 2014) even in the absence of exact concepts of the flow in the wake. As a result, the proposed methods of calculation were limited to a different set of assumptions and all in all did not meet expectations on their application for the development of the theory of wind turbines interaction and for verification of numerical modeling.

Successful experimental investigation of new scientific problems posed by industrial wind energy is practically impossible in full-scale wind turbines in the wind farm. Primarily, this is due to the lack of reliable measurement techniques for studying a complex vortex system of wakes at large distances behind the rotors of wind turbines under real atmospheric conditions. In-situ atmospheric measurements are now limited to determining the wind velocity by contact sensors at the measuring masts near the wind farm or non-contact laser velocimeters (lidars, etc.) that are less reliable at great distances. The first ones allow estimating the velocity profiles in a fixed position in space, along the mast, and the second ones measure some averaged velocity values at different random points, but with a large error and not simultaneously, i.e., in different time intervals for subsequent averaging. Under undetermined atmospheric pulsations, the obtained information on average values spread in time and space becomes uninformative for subsequent analysis and generalization. So, today the priority role in the experimental study of rotor interaction in FW still belongs to laboratory model studies.

Physical (laboratory) modeling in the development of rotor aerodynamics has always been an effective research tool, since its formation in the early twentieth century. At that time, the Russian aerodynamic school of Professor N.E. Joukowsky, stimulated by the progress of aviation, held leading positions in the world in the creation of rotary aerodynamics (Kuik et al., 2015; Okulov, Sørensen, Wood, 2015). Today the interest of researchers has shifted from aviation to the problems of wind energy, industrial development of which has been still objectively delayed in Russia. Despite the obvious production lag, the Russian scientists in their scientific research and development continue to obtain world-class results in this field of knowledge. This is facilitated by two factors: the first, of course, is a huge theoretical groundwork, created previously by the Russian school, and the second is problems with the accuracy of field measurements, necessitating the development of fundamentals of aerodynamic interaction of wind turbines in the wind farms on the basis of the available laboratory facilities. The current state of the studies results from to the lack of high-precision field measurements. The lack of reliable field data has been compensated for the use of both domestic and imported contactless high-precision laboratory measuring equipment in the model experiments. 
It served to reveal important new patterns and to build theoretical models of aerodynamic rotor interaction for optimizing the FW, which have made a significant contribution to the development of the classical problems of rotor aerodynamics.

The aim of this review is to consider the role of modeling in the development of rotor aerodynamics and to describe the most important results obtained in the last few years.

\section{The role of simulation in the development of rotor aerodynamics}

Theoretical studies on the rotor aerodynamics (marine propeller, propeller, wind turbine, etc.) have always been accompanied by a simultaneous development of experimental modeling. It should be noted that the first experience of such modeling was not very successful. It was associated with testing the theory of the loaded disk - the simplest and the oldest mathematical model of the propeller. In this abstract model, the load is replaced by a uniform distribution of the pressure jump on the infinitely thin water-permeable disk, replacing the rotor-swept area. The idea of such replacement dates back to the work of Rankine (Rankine, 1865). A strict mathematical proof of the loaded disk action was found by Froude Jr. (Froude, 1889), who showed that this theoretical model of the propeller/turbine has to accelerate/decelerate the flow by one third before the disk, and by another third after it, with a significant contraction/expansion of the wake behind the rotor for optimum operation (the middle fragment, Fig. 1a).

Unfortunately, the experimental modeling at first did not confirm this simplest model of an ideal propeller or turbine and led to its wrong interpretation, stating that behind the rotor the fluid can neither speed up nor slow down, but flows in the form of an unperturbed cylindrical column. Such misinterpretation was based on Parson's experiment, described in the comments to the article (Froude, 1911). In fact, a nonexpanding or very slightly expanding wake may be observed at partial loads on the rotor (Fig. 1b), and at normal or accelerated rotor work, the wake expansion is well visualized (Fig. 1c). Perhaps, in the Parson's experiment, the rotor worked at part-load, or a blocking effect appeared in the setup (Segalini, Inghels, 2014), distorting the flow behind the rotor. The misinterpretation of this experiment resulted in a lag in the development of the rotor theory in England compared to the success of the Russian and German scientific schools (Kuik et al., 2015).

In the early twentieth century, the first wind tunnels or water channels used simple experimental methods and techniques: air visualization of the vortex structure behind the propeller in the water, and determination of flow heterogeneities due to the deviation of paper strips or filaments in the wake behind the rotor in the air. These were Flamm visualization (Flamm, 1909) behind the propeller (Fig. 2a) and fixation of flow heterogeneity behind the propeller by D.P. Ryabushinsky that formed the basis of N.E. Joukowsky's reasoning of the creation of the vortex theory of the marine propeller (Joukowsky, 1912). Successful interpretation of these model experiments enabled the formulation of the vortex theory of the marine propeller (Fig. $2 b$ ) seven years before the German aerodynamic school of L. Prandtl (Betz, 1919).

The vortex model of the rotor is now the main tool to study air and water propellers, wind turbines, compressors, and turbines (Okulov, Sørensen, Wood, 2015). Moreover, it is the moldel that allowed showing the faultiness of the Parson's experiment and proving the correctness of the Froude theory. Helical structure of tip vortices generates additional acceleration/deceleration of the flow in the wake behind propeller/turbine, which increases the flow deceleration (Fig. 2c), associated with the selection of kinetic wind energy in the plane of the rotor. 


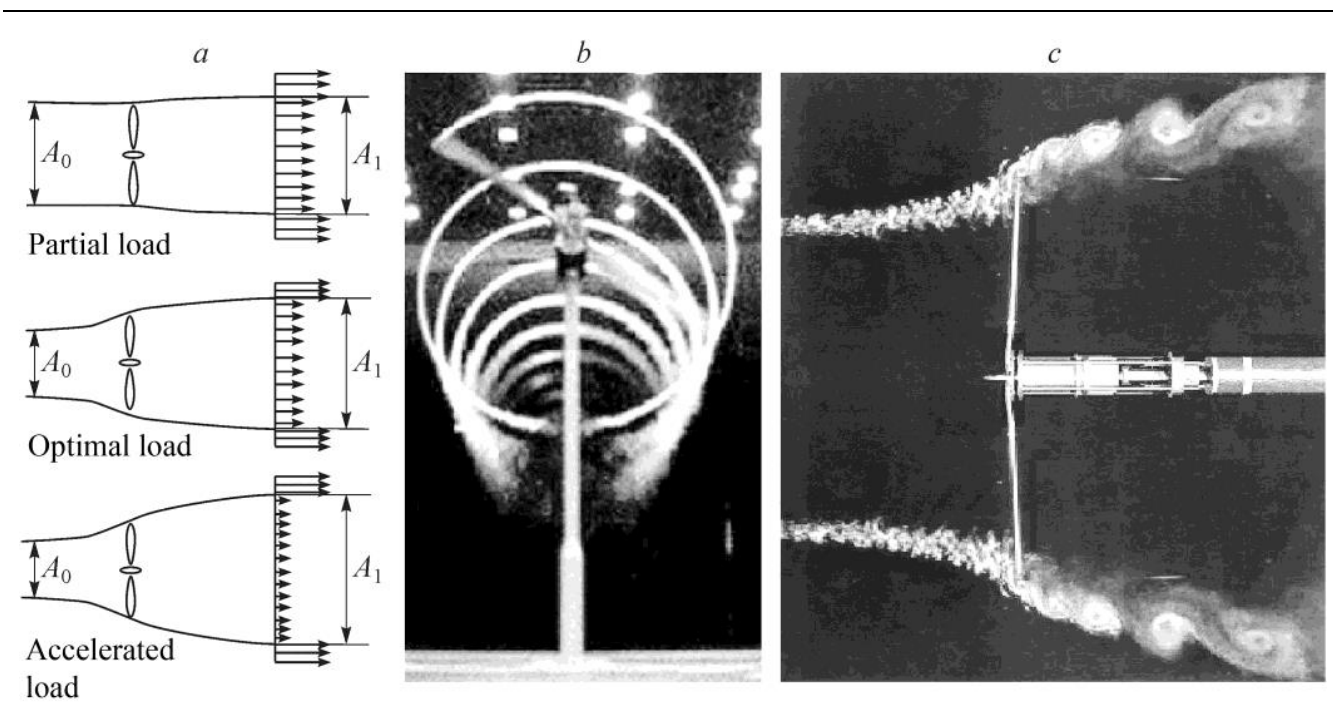

Fig. 1. Shapes of the wake behind the rotor.

$a$ - schemes of flow development according to the jet theory of the loaded disk

for different operation modes of the turbine (Hansen, 2008),

$b$-visualization of the mode of partial load without wake expansion (Hand et al., 2001),

$c$ - visualization of the optimal operation mode of two-bladed rotor with flow tube expansion (Holten, 1981).

131

At optimum modes of the turbine operation, the deceleration doubles, which fully confirms the Froude theory.

The demand for laboratory testing today is due to a complex vortex structure of swirling flows behind the rotating impeller, requiring specific methods and techniques of research using modern high-precision measuring equipment (Okulov et al., 2007). In the wind energy, the more accurate measurements provide laboratory testing with a distinct advantage over in situ (field) measurements. As noted previously, the direct use of field tests is limited by the lack of reliable diagnostic tools for atmospheric currents. At large distances in FW it is possible to fully study only the average flow characteristics in front of or in the wake behind the wind turbine rotor (Larsen et al., 2007), but the important information about the coherent helical vortex structure of the wake, which is well-fixed by visualization in the laboratory conditions, is lost with averaging (Figs. 1 and 2). Recently, the existence of a wake structure with helical tip vortices behind a full-scale wind turbine was effectively demonstrated in the unique full-scale experiment (Hong et al., 2014), carried out under special conditions. Thus, laboratory testing continues to play a decisive role, as for laboratory scale there are well developed measurement technologies that have been successfully used (Okulov et al., 2007).
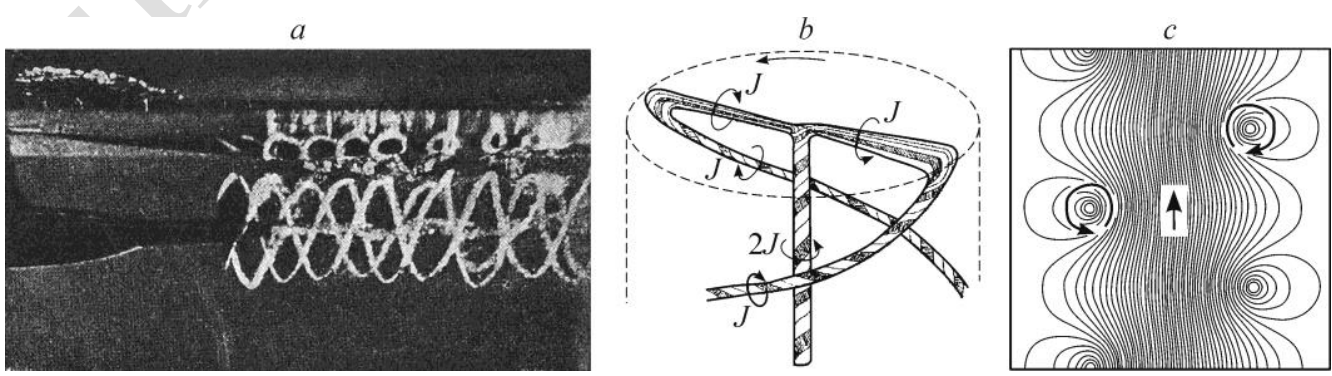

Fig. 2. Helical vortex structures.

$a$ - visualization of the vortex structure behind the ship propeller in water (Flamm, 1909), $b$ - scheme of vortex model of rotor-propeller (Joukowsky, 1912), $c$ - structure of the flow induced by helical vortexes, longitudinal section (Alekseenko et al., 2003). 
Another argument in favor of the need for preliminary laboratory studies is associated with separating the extraneous and external effects and eliminating the uncertainty of unique atmospheric conditions of the experiment, which gives the opportunity to test different models of rotor aerodynamics and to identify patterns of the rotor wake distribution. Laboratory modeling in many cases is preferable to a direct field experiment, as it allows repeatedly reproducing the same inlet conditions on the rotor for the statistical set of data needed for processing with a high degree of realizability. Therefore, it is advisable to start research on rotor interaction in FW from model experiments. Under in situ conditions, there are unpredictable effects on the rotor and the wake mainly due to temporal and spatial non-uniformity of wind loading and large-scale turbulence of the atmospheric boundary layer. In addition, currently because of the growth in wind turbine size there are additional effects in blades streamlining. For example, in their upper position, the wind velocity can reach $100 \mathrm{~m} / \mathrm{s}$, and to describe the aerodynamic characteristics it may require consideration of compressibility, temperature stratification and icing (Sunden, $\mathrm{Wu}, 2015$ ) etc. In varying degrees, all of these perturbations are important, but they are still secondary factors affecting the operation of wind turbines and complicating the classical description of inter-rotor interaction, directly associated with the impact of rotors and vortex wakes, generated only by the rotating blades of preceding turbine. From the first half of the last century after the establishment of the foundations of classical vortex theory of the propeller, in connection with an increase in flight speed and alti-tude, similar factors of additional impact on the aircraft propellers have been taken into account in the form of corrections. By analogy with the aviation development, for FW, the account for additional impacts should become the next step after the establishment of the main regularities of wake development and principles of their interaction with the subsequent turbine rotors. All additional factors should be studied after finding the basic patterns. They should be viewed as perturbations of the initially established base models in order to assess the degree of each factor influence and to identify the need to take it into account or neglect it in "in situ" tests.

Another important question is the medium of modeling. For experimental verification of the basic models and for the development of new models of the rotor in the early twentieth century, in the period of aerodynamics formation, both air and water laboratory experiments were quite successfully realized. In the modeling of low-speed propellers and turbines (including wind turbines), the application of two different media is justified by the neglect of compressibility due to small values of the Mach number. Even at velocities of $100 \mathrm{~m} / \mathrm{s}$, the correction for compressibility, which is proportional to the square of the Mach number, does not exceed $9 \%$. However, sometimes there is unreasonable skepticism about modeling on water for wind turbines due to significant differences in densities and viscosities of these media. Indeed, locally there may be some differences, directly connected with solid streamlining and boundary layer formaiton, but for a wide range of Reynolds numbers, their influence on the wake formation behind blunt bodies is virtually absent (Sumer, Fredøse, 2006). Recently this fact was confirmed in the wakes behind the rotating rotors, where in the experiments fluctuations were discovered at close Strouhal numbers both in wind tunnels (Medici, Alfredsson, 2006) and in water channels (Chamorro et al., 2013; Okulov et al., 2014). The result in this case depends on the correct scaling of the studied phenomena that has been repeatedly confirmed by comparison of similar data obtained in different media. For example, in the works of N.E. Joukowsky (Joukowsky, 1912; Joukowsky, 1914; Joukowsky, 1915; Joukowsky, 1918), the vortex theory of the propeller was created based on Flamm visualization behind the propeller in water, and was verified by experiments of D.P. Ryabushinsky with air propellers. To study the rotor interaction of modern wind turbines the laboratory modeling in water channels (Fraunie et al., 1986; Chamorro et al., 2013; Neary et al., 2013; Okulov et al., 2014; Bachant, Wosnik, 2015) is often used for two reasons. First, for water it is easier to keep the characteristic Reynolds number at small-scale simulation of wind turbines in small working sections, limited by geometric sizes of wind tunnels (Treuren, 2015) and water channels. Secondly, visualization and diagnostics of distributed characteristics in water have been 


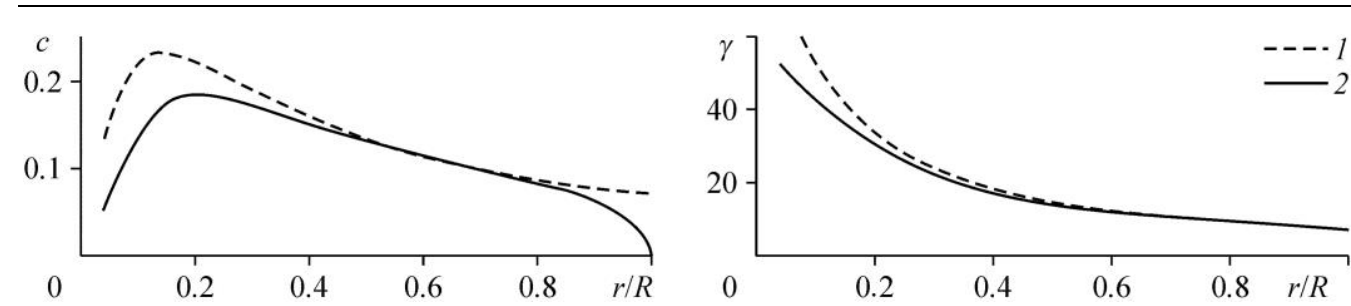

Fig. 3. The distribution of the chord $c$ and the local pitch distributions $\gamma$ for three-bladed rotor, built on BE/M method without adjustment for tip correction (1) and

for exact solution on BE/LL-method with Goldstein circulation (2).

worked out much better and give more accurate results. Further, we will demonstrate several examples of such modeling and diagnostics for water that were successfully implemented with the participation of the author of this review. In the described studies, the identity of the obtained results was confirmed by their comparison in the two media.

\section{Comparing the rotors of wind turbines constructed using different optimization methods}

The possibility of constructing the rotor blade through its separate elements cut with cylindrical sections, was considered by Drzewiecki in 1892 (Okulov et al., 2013). Using the proposed method, and in addition, to simplify the calculations, assuming that different flow tubes in each section behave independently from each other, Glauert calculated the parameters of the optimal rotor of the wind turbine (Glauert, 1935). He optimized equations of the pulse theory independently in each cylindrical flow tube, passing through the circular cross section, for any fixed value of the rotor radius, neglecting an existing interaction between them and the pressure changes in the radial direction. A method of "blade element/momentum" (BE/M) with different types of corrections for end effects is still the main tool in the design of rotors of wind turbines (Kuik et al., 2015). However, for modeling the blades, there is another vortex approach (Okulov, Sørensen, Wood, 2015). It is based on "blade element/lifting lines" (BE/LL). To determine the optimal distribution of circulation in BE/LL Goldstein (Goldstein, 1929) applied the variational principle of Betz (Betz, 1919). In the rotor with such blade, or for short - in the Betz rotor, the distribution of circulation along the lifting line, replacing the rotating blade, shall provide for the coming down free vortex sheet to have a strictly helical form and to move uniformly in the axial direction. By analogy with the elliptic wing, such field of free vortices in the wake behind the rotor must meet its minimum inductive resistance and correspond to its optimum operation mode (Okulov, Sørensen, Wood, 2015).

In order to verify and compare the efficiency of rotors designed using different approaches (BE/M and BE/LL), a pair of identical three-bladed models, built on the original BE/Mmethod without correction for end effects and on the exact solution of the BE/LL-method with Goldstein circulation was experimentally compared (Okulov et al., 2015). The algorithm was developed for determining the form of blades for two rotors with the same impeller diameter $2 R=0.376 \mathrm{~m}$ for the value of tip speed ratio $\lambda=5(\lambda=\Omega R / V$, where $R$ is the radius of the rotor, $\Omega$ is the angular velocity of its rotation, and $V$ is the free-stream velocity). Changes of the chord $c$ for SD7003 profile (Selig et al., 1995) and a final angle of inclination in each section of the blades with a correction for the same angle of attack $\alpha=4^{\circ}$ along the blades are shown in Fig. 3. In the design of the two blades, the influence of the rotor hub was neglected. For both rotors, it was the same, and this consequently led to the same influence. Note, however, that the question of the influence of the hub size on aerodynamic characteristics of wind turbines was studied separately. In the framework of the conducted research, it was established that for small hub ratios, typical of wind turbines, the influence of the hub on the aerodynamic characteristics of the rotor is negligible (Okulov, Sørensen, Shen, 2016), which is consistent with the conclusions made earlier at optimization of propellers (Wald, 2006). Both rotors were 

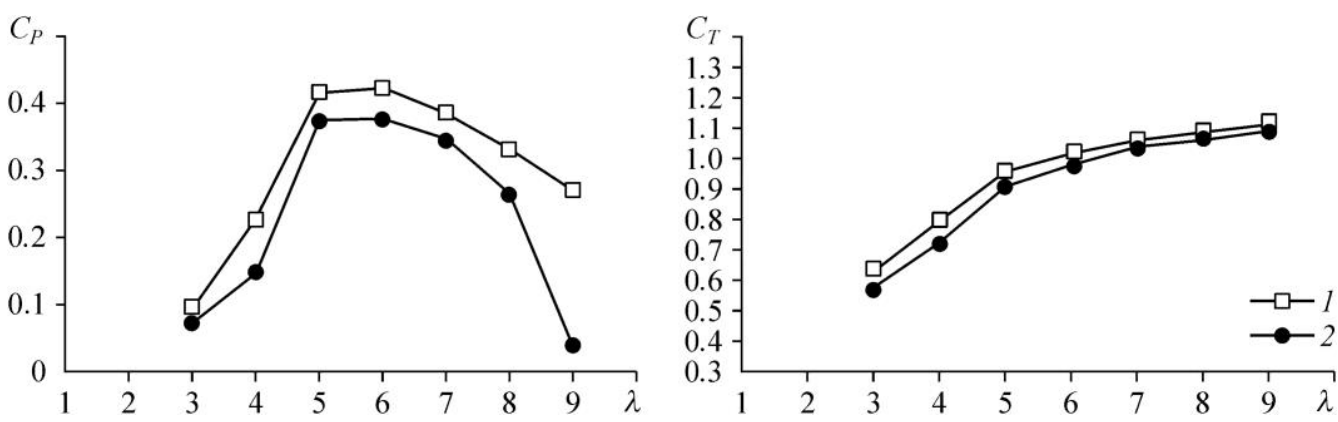

Fig. 4. The coefficients of power $C_{P}$ and thrust $C_{T}$ for Betz (1) and Glauert (2) rotors as a function of tip-speed ratio.

experimentally investigated in a water channel in order to compare their performance depending on the tip-speed ratio. The question of the suitability of water channel or wind tunnel in this case was not relevant, since the experiment only meant to test two different philosophies of optimal design of the turbine blades, i.e., it was enough to compare different shapes of rotors, ensuring equal conditions of testing. Both models were placed in the channel at the operating temperature of $20^{\circ} \mathrm{C}$, the Reynolds number of about 240000 , and the value of the oncoming flow velocity at the rotor location $U_{\infty}=0.65 \mathrm{~m} / \mathrm{s}$ with its fluctuations not exceeding $3 \%$. Power characteristics of the model rotors were measured by strain gauge sensors, installed at the rotor mounting. Measurements were made for flow torque and thrust on the rotor for tipspeed ratios $\lambda=3-9$. Figure 4 shows the corresponding dependences of power factors $C_{P}$ and thrust $C_{T}$ for both propellers. The maximum performance $C_{P_{\max }}$ of both rotors was achieved at

design tip-speed ratio of 5 and keeps its value up to $\lambda=6$ (Fig. 4). For the first time, it was found that the three-bladed rotor of Betz built exactly on the vortex method BE/LL allows obtaining more kinetic energy from a uniform incoming flow.

Along with the experiment, a comparison of the shapes of blades took place; some of them were obtained by calculation as a result of applying BE/LL-method and others were obtained by other designing approaches, including consideration of the continuous distribution of circulation along the blades - NEJ rotor (Joukowsky, 1912; Joukowsky, 1914), and the limiting cases of infinite number of blades. More detailed information about various models may be found in reviews (Kuik et al., 2015; Okulov, Sørensen, Wood, 2015), as well as in the book (Sørensen, 2016).

The works (Sørensen, 2016; Sørensen et al., 2016) compared the shapes of the blades, constructed analytically in accordance with different theories. Figure 5 shows the results of
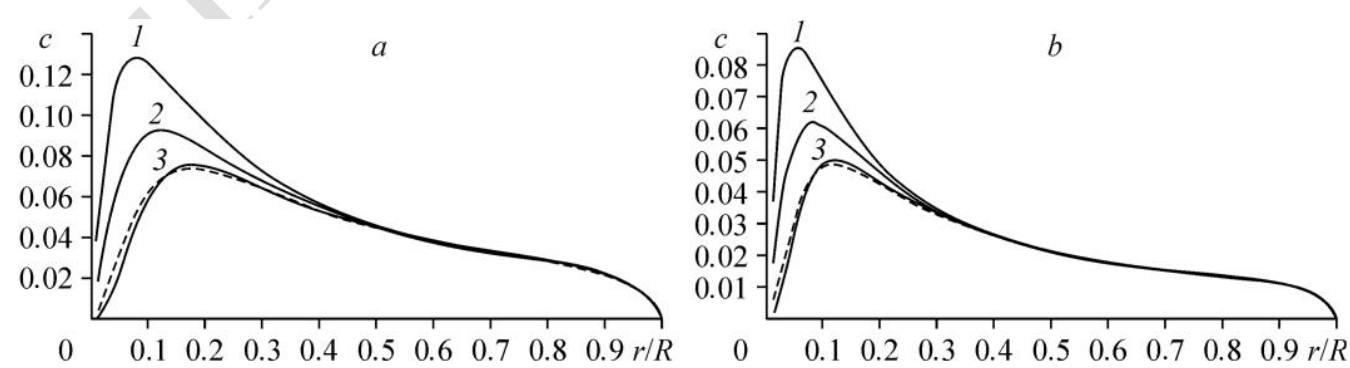

Fig. 5. The change of the chord $c$ along the blade $r / R$ for tip-speed ratios $\lambda=6(a), 9(b)$ in the calculating of the balde shape by different simulation methods.

Solid lines - approximations on model with an infinite number of blades with correction for the number of blades on Prandtl correction (Sørensen, 2016):

$1-\mathrm{BE} / \mathrm{LL}$ with constant circulation NEJ, $2-\mathrm{BE} / \mathrm{M}, 3-\mathrm{BE} / \mathrm{LL}$ with Goldstein circulation; dashed line - accurate calculation on BE/LL for three-bladed rotor of Betz (Sørensen et al, 2015). 
comparison of different geometries of the blades, constructed with different approaches for turbines with different tip-speed ratios. For a more correct comparison, the correction for the end effect of the blade was applied to approximate models with infinite number of blades. The effect of different methods of correction of the blade tip shape, starting with the first correction proposed by Prandtl (Betz, 1919), was investigated in the work (Wood et al., 2016).

Figure 5 shows that the blades designed by BE/M or different approximations on BE/LL method, after additional end correction of Prandtl, model the blade tip in very good agreement with the Betz rotor, constructed on the exact BE/LL solution for a finite number of blades and Goldstein circulation. The comparison shows that the geometry of the blades depends on the calculation method, but the differences mostly exist in the axial part of the blade and become significant for small values of a turbine tip-speed ratio, limited by value of 5 . The design of an optimal blade was further refined by taking into account the nonlinear influence of tip vortices (Wood, Okulov, 2017). As a result, a negligible impact of this additional non-linear correction on baseline BE/LL solution was found; moreover, it arose in a very narrow range for even smaller values of tip-speed ratios from 0.8 to 1.5 . Note that wind turbines typically operate at much greater tip-speed ratios, starting from 5 and above. Therefore, approaches to the construction of the blades, based on ideal models with an infinite number of blades in combination with the use of corrections for end effects (Wood et al., 2016) lead to practically identical geometries of the end blades. They are still very close to the shape of the blade, defined by the exact BE/LL solution for Betz rotor with a finite number of blades (Okulov, Sørensen, 2008; Okulov, Sørensen, 2010b). This important conclusion partly explains why the earlier practice of using approximate models in the design of the blades was so effective. However, the question on the role of differences in axial forms of blades on the performance of wind turbines remains unanswered yet. The most significant is this difference for Betz and NEJ rotors (Fig. 5, curves 1 and 3), and further investigation requires an additional experimental testing. Interest in this issue is warmed up by the discussion about the possibility to obtain a larger kinetic energy of the wind by NEJ rotor compared with the Betz rotor; it is based on analytical study of different limiting models with an infinite number of blades for small tip-speed ratios (Sørensen, Kuik, 2010). Recent theoretical study (Kuik, 2016) once again established the superiority of the NEJ rotor, but again, in an unrealizable ideal limiting case with infinite number of blades. The answer can be obtained only after experimental comparison of the Betz and NEJ rotors with a finite number of blades.

\section{Studying the wake behind the disk in water channel and wind tunnel}

Before proceeding to the modeling and study of the development of rotor wakes, let us investigate the properties of self-similarity in the wakes behind bluff bodies, in this case behind the disk in different media: in air and water ones. To describe the wake behind the disk in water such testing was conducted in the work (Naumov, Litvinov et al., 2015). With the help of measurements made by digital tracer visualization (PIV) and laser Doppler anemometer (LDA), the regularities of attenuation of the axisymmetric turbulent wake behind a stationary disk in a water channel were investigated, and a comparison with the experiments performed earlier with the comb of thermoanemometers behind the stationary disk in a wind tunnel was realized (Johansson, George, 2006). In a turbulent axisymmetric wake behind the disk, the property of self-similarity (self-similarity of velocity profiles) was tested; it has to manifest and should be sustainably reproduced in a wide range of Reynolds numbers, covering the regimes of operation of real wind turbines. It seemed interesting in each cross section of the wake behind the disk at a distance $x$ for different media to compare the form of dimensionless profiles of velocity deficit $\left(U_{\infty}-U(r)\right) / U_{0}$, where $U_{\infty}$ is the freestream velocity, $U_{0}=\max \left(U_{\infty}-U(r)\right)$ is the velocity deficit on the wake axis, and $U$ is the profile of the longitudinal velocity 
Thermophysics and Aeromechanics, 2018, Vol. 25, No. 1
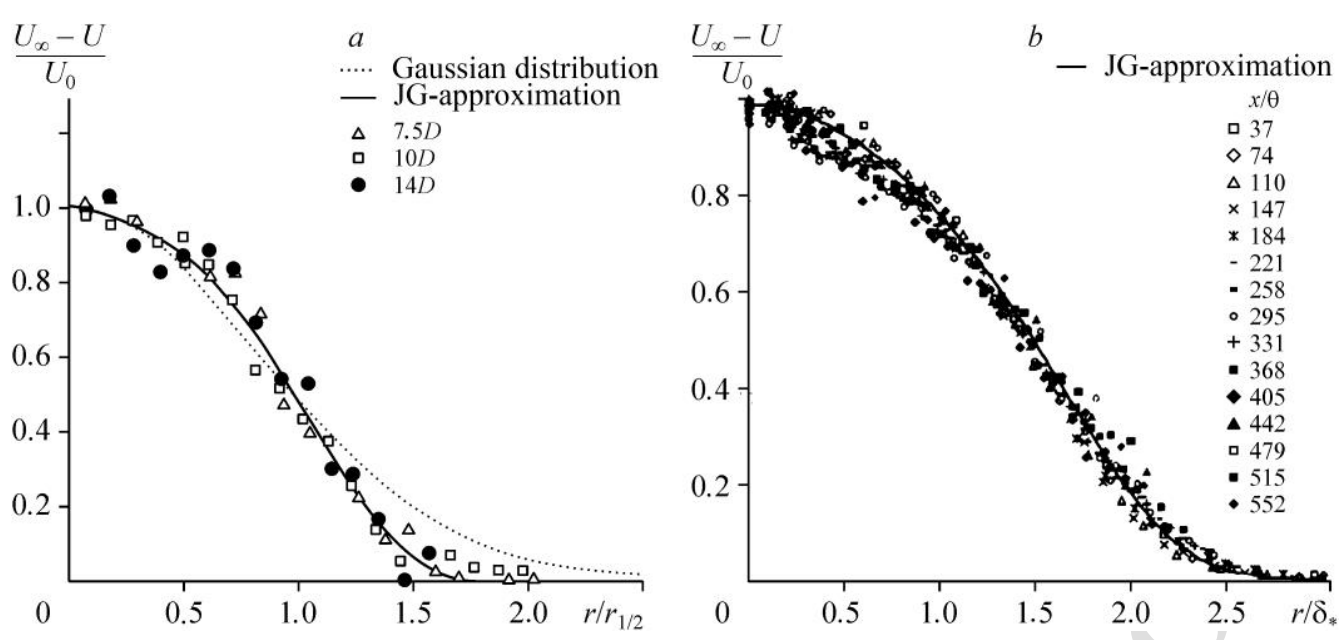

Fig. 6. Testing self-similarity of velocity profiles in the wake behind the disk

$a$ - water channel with profile width normalization at the point of half-value of the deficit (Naumov et al., 2015), $b$ - wind tunnel normalized by the profile width (Johansson, George, 2006).

component. For profile description, the JG-approximation $\left(1+a r^{2}+b r^{4}\right) \mathrm{e}^{\left(-c r^{2}-d r^{4}\right)}$ with fixed parameters $a=0.049, b=0.128, c=0.345$, and $d=0.134$ proposed in (Johansson, George, 2006) (Fig. 6b, the distance between the profiles and the disk $x / \theta=552$ corresponds to $150 D$ ) was used. JG-approximation differs slightly from the usual Gaussian distribution, but better models the wake in both tests (Fig. 6) both behind the disk in the water channel and behind the disk in the wind tunnel, for small Reynolds number $\operatorname{Re}=2 \cdot 10^{5}$ and $\operatorname{Re}=2.6 \cdot 10^{4}$, respectively, falling within the range of existence of the self-similar wake.

Another important issue is to clarify the influence of the medium phase state on the attenuation of the velocity deficit in the wake along the disk axis downstream. This attenuation behind the disk in the water channel coincided with a rational dependence $0.32(x / D-3.2)^{-2 / 3}$, established in earlier experiments in the air in the wind tunnel (Johansson, George, 2006) (Fig. 7). The Figure shows that at points of the far wake $(x / D \geq 5)$, there is a monotonous decay of deceleration with a degree of $-2 / 3$ approaching the asymptotic horizontal line at $x / D \geq 25$, which determines the level of background fluctuations in the oncoming flow below which the existence of a wake is impossible to distinguish. Note that the deceleration rate of the wake behind the disk in the water channel coincided with its attenuation behind the disk in the wind tunnel despite the above differences in Reynolds numbers. Indeed, for a wide range of relatively large Reynolds numbers, the degree of $-2 / 3$ for turbulent axisymmetric wake attenuation remains behind any blunt axisymmetric body (George, 1989; Novikov, 2009). For small, subsonic flow velocities it does not depend

Fig. 7. A comparison of attenuation of velocity deficit behind the round disk in the water channel and the wind tunnel. 1 - a power dependence with exponent of $-2 / 3$,

2 - measurement in the air (Johansson, George, 2006), measurement in water:

3-PIV technique, 4 - LDA method:

5 - the level of external disturbances In the water channel (Naumov et al., 2015).

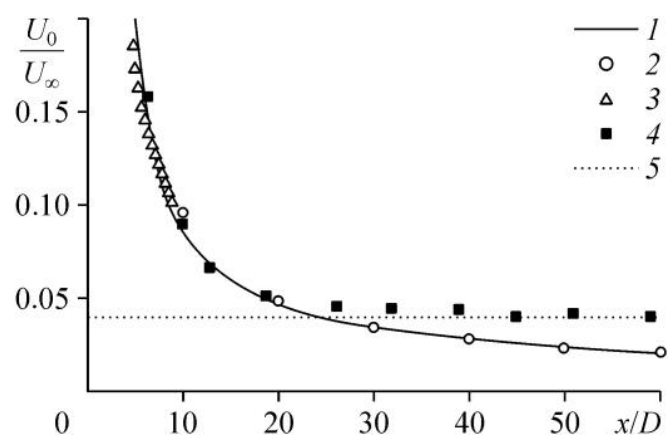


on the medium (water or air). In addition, it has been recently discovered that the degree of attenuation of the wakes remains at their non-axisymmetric streamlining as well (Jiang et al., 2016). Data presented in the paper (Naumov et al., 2015) confirm the universal properties of self-similarity and attenuation of a far turbulent wake at relatively high Reynolds numbers and the possibility of their studies in water channels and wind tunnels.

\section{Comparing the wakes behind the rotor and the disk}

The prediction of the operation mode of the wind turbine falling in the wake from the previous turbine, is fundamental for calculating losses and optimizing the wind farms. To solve this problem it is necessary to create an adequate model of the wake development behind the rotor. Today, the most popular are the kinematic models based on self-similarity profiles of velocity deficit at the conservation of mass and momentum in the wake, which use the assumption of a linear wake expansion and the experimental value of the coefficient of flow thrust on the swept surface of the wind turbine rotor as input data. It should be noted that the assumption of a linear wake expansion and conservation laws uniquely determine the wake attenuation according to the hyperbolic law with the exponent of -1 , which differs from the above considered case of its attenuation behind the blunt bodies with index $-2 / 3$. Although there has been no purposeful experimental study of wake development behind wind turbines for sufficiently large distances, a priori applied in practice is the model of Jensen (Jensen, 1983 ) with wake attenuation coefficient of -1 or its modifications, specifying only the shape of the velocity deficit profiles in the wake. For example, in (Bastankhan, Porte-Agel, 2014), it was proposed to use a Gaussian distribution. The next step of the research was a more thorough examination of the law of deceleration of the far wake behind the rotor.

An overall idea of the flow structure behind the rotor operating in turbine mode has been long known. Taking the energy of the incoming flow, the turbine rotor slows it down, forming a characteristic velocity gap in the wake - the deficit characterisitic of the wakes behind bluff bodies. The fundamental difference between the wakes for blunt bodies (in our consideration, disks) and rotors should be noted as well. In the first case, the flow deceleration is due only to the resistance of the body and has a passive character. In contrast, the flow deceleration behind the rotor is a result of its active influence and is associated with kinetic energy removal from the flow. In the rotor plane, the flow is decelerated by the useful energy removal. At optimal mode, the deceleration in it is about $1 / 3$ of the initial velocity, but it is not finished and further occurs already in the wake. The velocity is additionally reduced by one more third, and total deceleration reaches $2 / 3$ of the unperturbed velocity.

In the near wake, when the effect of the turbine on the flow is over, additional braking is determined by the impact of the system of helical vortices (Fig. $8 a$ ), coming off the ends of the blades. They generate one more flow along the axis (Fig. 2c), which further slows the flow. Such flows do not exist in the wake behind the disk (Fig. 8b). Behind the rotor, such additional deceleration is registered in all the dimensions of velocity profiles of the near wake (Naumov et al., 2012; Naumov et al., 2014). Its growth completely stops at a distance of 2-4 diameters of the rotor, when the vortex system is completely destroyed and its effect is over.

Additional deceleration of the averaged velocity profiles in the near wake was also repeatedly registered in different field experiments (Vermeer, Sørensen, Crespo, 2003). However, there was some skepticism about the possibility of using data of model studies on the influence of helical tip vortices on the formation of the near wake behind real wind turbines, because their existence could not be fixed in situ by any available means of measurements in the atmosphere (Larsen et al., 2007). The authors of (Hong et al., 2014) undertook a unique field experiment, where the original solution, namely the use of snowflakes for the tracers in large-scale PIV-measurements in the near wake behind a $2.5 \mathrm{MW}$ wind turbine, served to clearly determine the existence of concentrated tip vortices, predicted in many air and water model experiments. 
Thermophysics and Aeromechanics, 2018, Vol. 25, No. 1
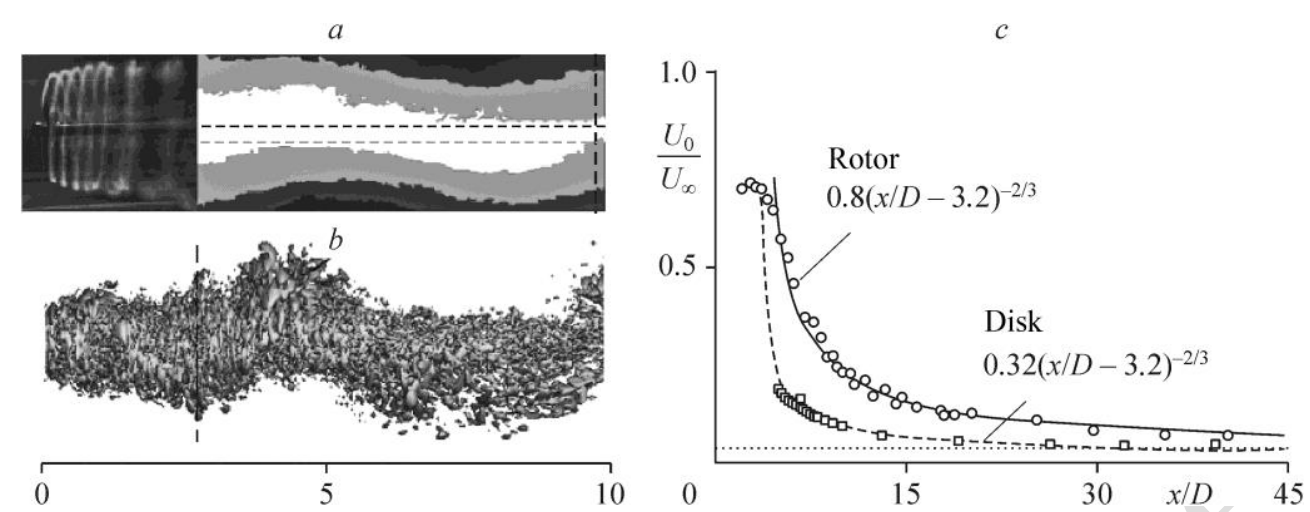

Fig. 8. Comparison of the wake structure

The wake over the active rotor (Okulov et al., 2014) (a), passive disk (Yang et al., 2014) (b); a comparison of the attenuation of velocity deficit in both wakes (Litvinov et al., 2016) (c).

Termination of the effect of tip vortices in the wake is due to their destruction, as the full vortex system in combination with a central axial vortex (Fig. 2b) is absolutely unstable (Okulov, Sørensen, 2010a; Felli, Camussi, Di Felice et al., 2011). Behind the rotor at distances over its 2-3 diameters, helical vortices are destroyed (Fig. 8a) and their effect on the flow is stopped. Formation of the long wake starts after the destruction of the near wake and the maximum braking at a distance from 2 to 5 diameters from the rotor. Behind the near wake (highlighted area in Fig. $8 a$ and $8 b$ ), first, the far wake both for the rotor and the disk similarly forms into a large helix. The comparison of the dynamic characteristics of the wakes in this region has revealed the development of low frequency oscillations at constant Strouhal number, the well-known phenomenon at streamlining of a blunt body (in this case, the disk) that is reproducible in a wide range of Reynolds numbers. In addition, for the first time for rotor, the work by (Okulov et al., 2014) established a weak dependence of the frequencies of these oscillations on the parameter of tip-speed ratio of the rotors for optimal and accelerated loads. This is because at rapid rotation, the surface swept by the rotor acts similar to an impermeable disk at the forming of the shape and dynamics of low frequency oscillations of the far wake. Note that the presence of low frequency oscillations at Strouhal frequencies was established in the wakes behind the rotors for both media: in experiments in the air in the wind tunnel, this frequency was recorded in (Medici, Alfredsson, 2006), and a full-scale study in the water channel was conducted by the authors (Okulov et al., 2014). As a result, once again, the correctness of the modeling of the dynamic development of the wake in both media was confirmed.

In experiments (Okulov, Naumov et al., 2015), the similarity of the far wake behind the active rotor with the wakes behind the passive fixed blunted bodies, disk or sphere, was also observed in their further development. The purpose of the work (Naumov, Mikkelsen, Okulov, 2016) was the creation of an experimental model of the wake behind the wind turbine rotor to assess the maximum distance of its propagation. In Fig. $8 c$, a solid line shows a curve approximating a change in average axial velocity in the far wake of the rotor depending on the distance from it. The far wake starts forming after the destruction of the near wake and the reaching of its maximum braking behind the rotor at a distance from 2 to 5 diameters. Additionally, Fig. 8c shows a comparison of the above data with the data for the wake development behind a disk. Their comparison has shown that the attenuation of the far wake in both cases follows the same power law differing only in the intensity of velocity deficit in the wakes, which is reflected in the coefficients at equal power dependence of attenuation (Fig. 8c). In the wakes behind the rotor, this relative ratio of attenuation intensity has a much larger value (0.8) compared to the value behind the disk (0.32). The differences in the intensities of velocity deficit are understandable. Thus, in contrast to the passive flow deceleration 
417 behind the disk, the rotor actively influences the incoming flow, transforming its energy into torque, and, in addition, extra deceleration is generated by helical tip vortexes.

Note that the self-similar behavior of the far wake was independently established in different sections behind the rotor of the wind turbine in the water channel (Okulov, Naumov et al., 2014) and wind tunnels (Dufresne, Wosnik 2013; Bastankhan, Porte-Agel, 2014). The authors of the latter two works showed self-similarity of the wake behind the rotor, but due to an insufficient length of the measuring section, small values of the rotor tip-speed ratio and lack of precision of air measurements in these works, the values of the coefficients in the laws of attenuation were difficult to establish exactly. The data of the testing of selfsimilarity of the wake behind the rotor in the water channel (Okulov, Naumov et al., 2015), presented in Fig. 9, showed a good agreement of the shapes of profiles in the wake and JGapproximation proposed to describe the wake behind the disk by the authors of (Johansson, George, 2006) and previously used to analyze the data in Fig. 6. In addition, the authors (Okulov, Naumov et al., 2015) first discovered and described the presence of the same selfsimilarity for different tip-speed ratios of the rotor (Fig. $9 b$ ). The difference occured only for slow rotation of the rotor $\lambda=2$ (with a design optimum at $\lambda=5$ ), when the approximating function was closer to a Gaussian distribution rather than to JG-approximation.

Thus, the behavior of the far wake behind the rotor was to a large extent identical with its behavior behind the disk, starting from the occurrence of low-frequency oscillations at Strouhal frequency, weakly dependent on the change of the Reynolds number and ending with the wake self-similarity with the same degree of attenuation with exponent of $-2 / 3$, but with different intensity factors. At conservation of mass and momentum in the wake, this attenuation determines the power law of its expansion with exponent of $1 / 3$, i.e., the real behavior of the wake differs from the used kinematic models with linear expansion (Jensen, 1983; Bastankhan, Porte-Agel, 2014; Bastankhan, Porte-Agel, 2016). Consequently, the use of kinematic models with a linear expansion of the wake is not entirely correct and can probably only be successful at its short segments, where a linear approximation is performed for a wake really expanding according to a different law. One more simplified modeling may be realized, apparently, by determining the similarity of the behavior of the far wake behind the disk and
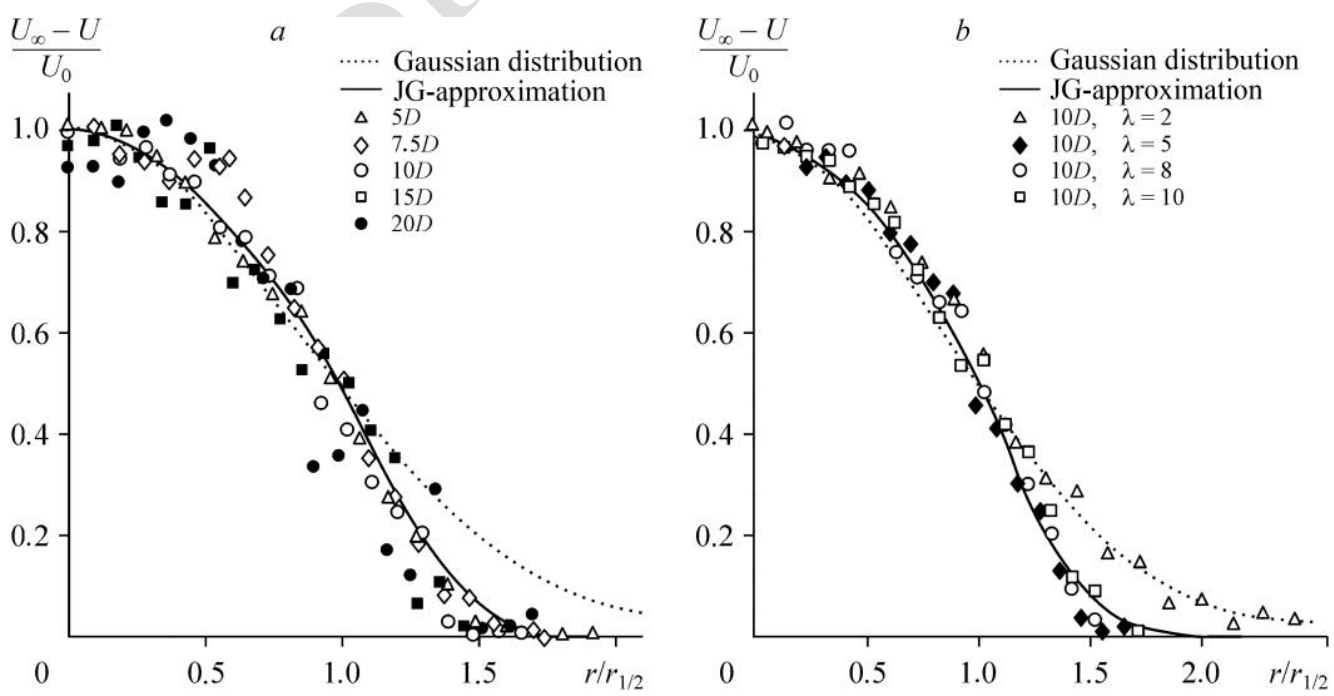

Fig. 9. Examining self-similarity of velocity profiles in the wake behind the three-bladed rotor depending on the distance behind the rotor $(a)$ and on its tip-speed ratio $\lambda$ (Okulov at al., 2015) (b). 
the rotor. For example, in the calculation of the wind farms for ease it would be convenient to replace the rotors by simpler disk models, using the formal correction of the wake intensity ratio from 0.32 to 0.8 or correcting the thrust coefficient, as in the model of Jensen (Jensen, 1983), but, of course, within the discovered power law of $-2 / 3$. These possibilities will be discussed in the next Section.

\section{The aerodynamic interaction of the rotors and disks}

Before describing the modeling results let us refer to the data of operation of the real different combinations of wind turbines, located in the wakes behind each other at frontal wind directions. In one case, it was expedient to choose two chains F20-F01 and J20-c J06 with wind direction of $312.2^{\circ}$ and spacing between turbines of $5.4 \mathrm{D}$ rotor diameters, and in the second, C19-M19 and A13-L13 with wind direction of $222.3^{\circ}$ and spacing of $8.3 \mathrm{D}$. Changes of the wind power removed by turbines along the chains in relation to the performance of the first turbine in the chain are shown in Fig. $10 a$ and $10 b$, respectively. The work of three chains of the four is characterized by the effect of stabilization of the energy removed from the wind or power produced by turbines. In the fourth chain (A13-L13), power is not stabilized but continues to fall from turbine to turbine, which is consistent with the classical idea of reducing the velocity of each turbine, at least by one-third, which naturally reduces the power of turbine falling in the wake of the next turbine and further throughout the chain.

Precise explanation of the stabilization effect in the turbine chain operating in the wakes of one another has not been found yet, although this effect is well known and is used in the operation of wind farms. The lines in graphs of Fig. 10 present the results of calculations on Jensen model at its local application and sequential transition from one turbine to another; every time the specifically measured thrust coefficient was used for the calculated turbine. Note that this step-by-step empirical correction describes well the effect of stabilizing the energy removed from wind by the wind farm that is expected due to correlation between generated power and wind thrust on the surface swept by the rotor, used as an empirical parameter of the model. That is the model of Jensen and its variants (Jensen, 1983; Bastankhan, Porte-Agel, 2014; Bastankhan, Porte-Agel, 2016) due to their empiric nature cannot clarify the reason for the stabilization effect, which is obviously associated with the recovery of velocity profile before the next turbine in the chain. In addition, these models do not
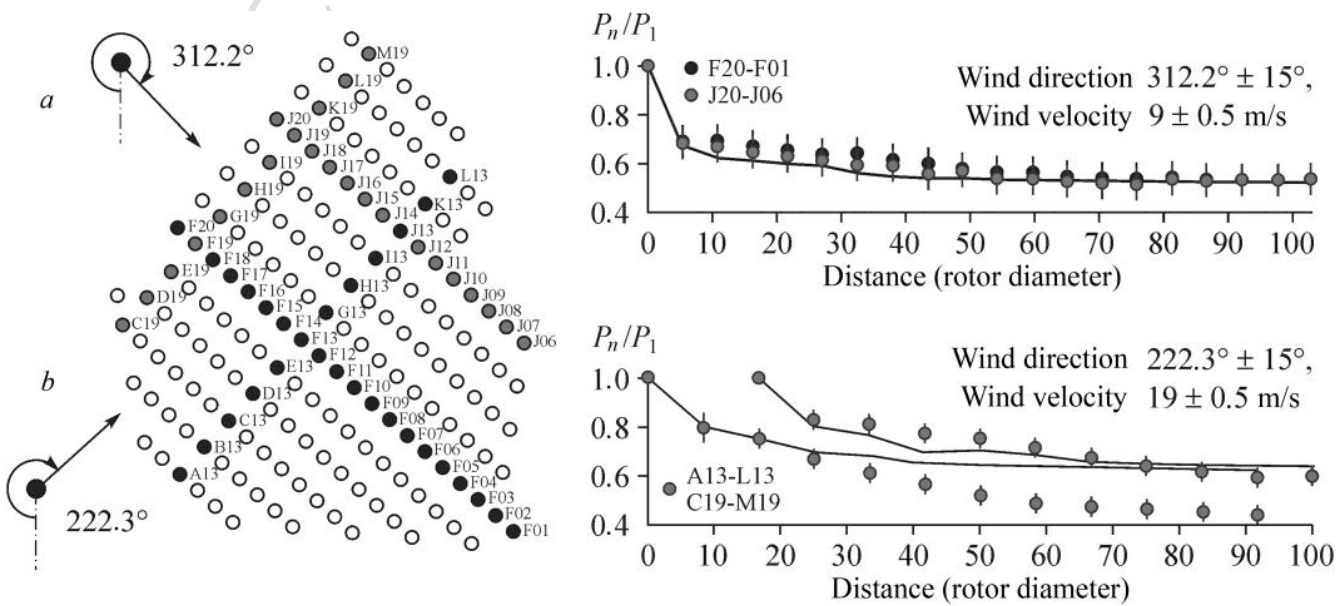

Fig. 10. Effect of stabilizing the energy taken from the wind in selected chains of wind turbines in the wind farm "London Array" (Nygaard, 2014). 
always accurately describe the actual operation of the wind farm (for example, for the mode in Fig. 10b). Here, significant are the remoteness of turbines in the wind farm providing a greater velocity deficit; the atmospheric turbulence responsible for more intense mixing; the nonuniformity of the velocity profile of the incoming flow or, more likely, the simultaneous impact of all factors affecting the recovery of the velocity profile in the wake. Of course, for a correct description of the phenomenon, it is useful to estimate the degree of influence of each factor on the studied effect, but, as mentioned earlier, today it is impossible to do in situ because of the lack of precision of the measuring techniques and the uncontrol-lability of natural conditions in the FW operation for eliminating certain factors. Thus, once again we return to the need for modeling, which can begin with the study of the interaction between two turbines in conditions of practical absence of external turbulent fluctuations and the nonuniformity of the profile of the incoming flow. Such an idealized modeling of working conditions will allow obtaining information about the basic interaction of the rotors to further add other disturbances and assess their individual impact on the system performance.

The study by (Okulov, et al., 2017) used two identical models of horizontal wind turbines (Fig. 11a). In sections 2 and 4, a single model of such rotor was used to study its strength characteristics and the wake behind it in the water channel. In the experiments, the rotor frequenices $n$ and the distance between them $H$ varied. Depending on the fixed values of these parameters, on the measured values, the power factor and the thrust coefficient were calculated for both rotors. It should be noted that determining the tip-speed ratio of the second rotor was more difficult compared to the first rotor since the velocity of the incident flow on the second rotor was not known in advance and could not be used in a definition of the tip-speed ratio. There were also problems due to uneven profile of the incoming flow velocity in accordance with JG-approximation of the wake behind the first rotor. However, the authors managed to establish a simple relation for estimating the maximum efficiency of the second rotor. It turned out that changing the power output of the second rotor, related to the first one's power, depending on the distance between them can be estimated using the law of wake attenuation behind a single rotor as a square of velocity change on its axis: $\left(U_{0} / U_{\infty}\right)^{2}=$ $=\left(1-0.8(x / D-3.2)^{-2 / 3}\right)^{2}$ (Fig. 11b). The figure presents not only the data of experiments in the water channel, but also the results of the experiments in the wind tunnel, which once again confirm the identity of modeling in different media. The latest data are represented by a single point because the dimensions of the working section of the wind tunnel did not allow making the distance between the rotors over $5 D$. In addition to the study of rotors arranged on the same
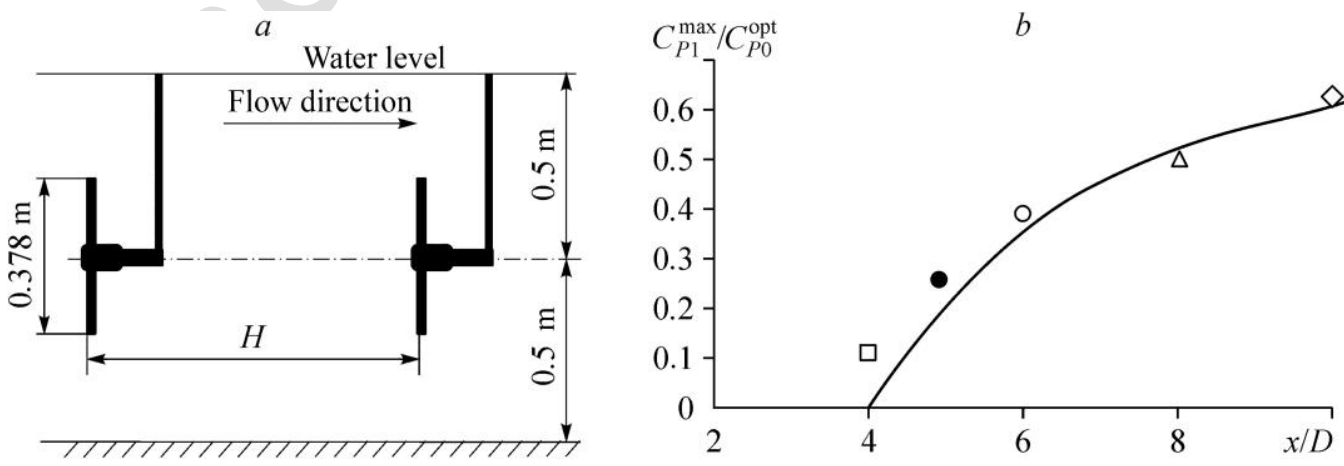

Fig. 11. The interaction between the two turbines.

$a$ - scheme of setup, $b$ - ratio of the maximum power of two rotors depending on the distance

between them $H=x / D$ : bright characters - experiments in the water channel (Okulov, et al., 2017),

dark character - experiments in the wind tunnel (Bartl et al., 2012);

solid line - the square of the relative velocity on the axis of the wake behind a single rotor. 
axis, the work (Okulov et al., 2017) studied the cases when the axes were shifted by half and by a full diameter. Nothing unexpected was found, and the worst was the case of rotors arrangement along the same axis when the second one fell completely into the wake of the first one.

Of particular interest is the study of the development of the far wake behind the second rotor and, accordingly, a comparison of its behavior with the wake generated behind two disks in the same configurations.

A comparison of the wake development at streamlining of passive systems with two immovable disks and behind two active rotors (Fig. 12a) has revealed a fundamental difference between them (Naumov, Litvinov, et al. 2016; Okulov, Mikkelsen et al., 2016; Okulov, Litvinov et al., 2017a; Okulov, Litvinov et al., 2017b). In particular, the introduction of the second disk in the wake of the first one causes an increased deceleration in a total wake, which is natural for passive systems and is related to the emergence of additional resistance of the second disk, increasing the initial braking and the total intensity of the wake. In contrast, the deceleration in the wake behind two active rotors decreases. A typical approach to explaining different features in the wake development is a reference to the difference in turbulence characteristics. However, in the considered experiments for all wakes, behind single and double passive and active systems, the distribution of the pulsations intensity was almost the same (Fig. 12b). Therefore, we can assume that the turbulent characteristics may not be the main reason of the established phenomenon.

The recorded difference in the behavior of the wakes behind passive and active systems is a crucial result, as the found decrease in the intensity of the wake behind the two rotors can become the key to unraveling the effect of stabilizing the power generated by the chain of wind turbines. To understand the unexpected fact, i.e., the decrease in the intensity of the wake behind two working rotors as opposed to its growth for two disks, let us recall the difference in the formation of the wake behind active and passive systems. As mentioned earlier, a strong influence on the formation of the wake behind the rotor is exerted by helical vortex system of tip helical vortices (Fig. 2 and Fig. 8a), providing an additional doubling of velocity deficit in the wake. It is reasonable to assume that the vortex system in the wake of the first rotor destroys the system of the second one, leveling its contribution and reducing the intensity of the total wake. Of course, this assumption should become the subject of comprehensive examination in further studies.

Indeed, the influence of large-scale pulsations in different wakes on the performance of turbines falling into them is not well understood yet. This fact is also confirmed by the study

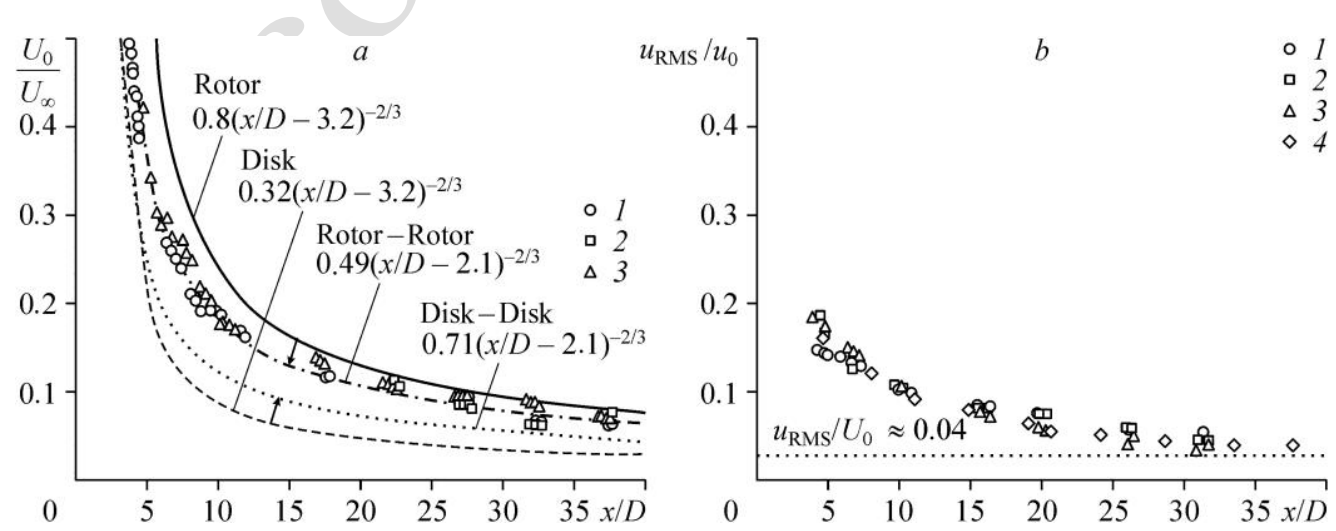

Fig. 12. Comparison of the far wake behind the single or double systems of rotors and disks.

$$
a \text { - velocity deficit attenuation: } H=4 D(1), 6 D(2), 8 D(3) \text {, }
$$

$b$ - development of turbulent pulsations (Okulov, Mikkelsen et al., 2016):

$L_{x}=4 D(R-R)(1), 6 D(R-R)(2), 8 D(R-R)(3), D, D-D\left(L_{x}=4 D-8 D\right)(4)$. 
of the effect of the wake from obstacles (disk) on the operation of the wind turbine (Naumov et al., 2016; Naumov et al., 2017). It is shown that at different positions of the obstacles relative to the axis of the rotor, the velocity deficit in the section in front of it ranges from 0 to $20 \%$, and the level of turbulence coming from the obstacle has always been high: from 8 to $15 \%$ compared to $4 \%$ of the background value in the free stream. It has been found that the capacity of wind turbine is affected only by its falling in the zone of the shadow from the wake behind the obstacle, and the increase in pulsations has almost no effect on the power level, which also requires a more careful study.

\section{Conclusion}

This retrospective review on the development of scientific bases of rotor aerodynamics (Kuik et al., 2015; Okulov, Sørensen, Wood, 2015; Fukumoto et al., 2015) has revealed the determinant role of laboratory testing in the study of the wind turbine operation. The role of these studies for modern solutions of wind power problems has been determeind. Return to laboratory testing at the present stage of development of rotor aerodynamics, as well as previously, is due to the impossibility of a full-fledged field study because of the complex nature of inter-rotor interaction in FW, the lack of sufficient precision and absolute irreproducibility of natural conditions, which substantially complicates common patterns and concepts in the field experiment. For the laboratory testing, it was necessary to analyze, adapt and further develop various precise methods for measuring the kinematic characteristics of fluid motion, using optical laser methods in the wakes behind the models of wind turbines with the dominance of helical vortex structures and their subsequent collapse and formation of the far wake. Using modeling it became possible to answer questions on the design of turbine rotors and to determine the regularities of the wake development and inter-rotor interaction. The effectiveness of different forms of turbine rotors based on various classical optimization techniques has been so far compared only at modeling. As a result, the larger performance has been revealed in the rotor designed in accordance with the concept of Betz (Okulov, 2014) and the distribution of Goldstein circulation along the blade (Okulov and others, 2015). It should be noted that various approximate methods of designing blade shapes with the use of different corrections on the impact of the blade ends (Wood et al., 2016) give good approximation to the shape of the optimal blade. With a slight influence of rotor hubs of small size (Okulov, Sørensen, Shen, 2016), these forms differ only when approaching the rotor axis (Sørensen et al., 2016), which indicates the high efficiency of existing wind turbines, the rotors of which were designed by various approximate optimization methods.

The priority fact discovered in the study of the development of the far wake behind the rotor is the detection of a uniform analytical model to describe its self-similarity and decay according to the power law with the exponent of $-2 / 3$ (Naumov et al., 2016). The identified model turned out to be suitable for arbitrary modes of the rotor operation in the modeling of the far wake formed in the breakup of the helical vortex structure of the near wake (Naumov et al., 2014). For different parameters of the wind turbine model operation, regardless of the structure of the near wake and forms of its collapse, the far wake always formed with the same structure, except for the modes of very small tip-speed ratio, not implemented in practice (Okulov, Naumov et al., 2015). The same structure of the far wake was produced in the case when the near wake consisted of inner and outer rings of helical vortices, descending simultaneously from hub and tip edges of the blades. The possibility of these modes to exist was seen repeatedly in experiments and was proved in theory as the existence of equilibrium states for internal and external helical vortices (Okulov, 2016).

An important stage in the presented review was to determine the influence of the wake of a preceding turbine on the performance of the following wind turbine. For distances between the turbines exceeding four calibers (on rotor diameter), a simple relationship has been found to determine the losses of the second wind turbine depending on its distance (Okulov, et al., 
2017). Of particular interest is the comparison of the behavior of wakes behind the separate rotor and the system of two rotors, arranged one behind the other. The recorded sudden decrease in the wake intensity behind the two rotors can be the key to unraveling the effect of stabilizing the generated power in the chain of wind turbines (Okulov, Naumov et al., 2016). Additionally, these experiments showed that the system of two disks can not satisfactorily replace the system of two rotors, for example, to simplify calculations, as in the first case, the total intensity of the wake increases, and in the second, decreases compared to a wake behind a single disk or rotor, respectively.

In the study of the influence of the wake of a large-scale obstacle, it was found that the capacity of wind turbines is affected by its presence in the zone of the wake shadow behind the obstacle, and the increased pulsations due to the presence of heterogeneity almost did not affect the operation of the wind turbine (Naumov et al., 2016). Certainly, further studies of the effects of different disturbing factors should be extended to stratified flows (Voropaeva et al., 2016) that model the natural non-uniformity of the atmospheric boundary layer.

The obtained results are undoubtedly of interest for the further development of the aerodynamics of wind turbines and wind farms, both to optimize the operation of existing FW, and to design and analyze the new ones. The findings formulated above in the description of the studied phenomena will be useful to improve the energy and economic efficiency, reliability and safety of impellers of axial turbines in wind power. In addition, the conducted research gives an impetus to new works related to further development of the FW. New research is necessary to complete the study on the selection of optimal shapes for the wind turbine blades. The question on the influence of the difference in the axial shape of the blades on the gas turbine performance should be answered in order to assess the theoretical solutions, predicting greater efficiency of the blades with constant distribution of circulation compared with the Goldstein circulation.

Important is the question about the influence of turbulent fluctuations of the incident flow on the efficiency of wind turbines. It is necessary to investigate the model setups at elevated levels of free-stream turbulence and compare them with the available data for almost turbulence-free mode. The study of the impact of various obstacles on the operation of wind turbines should be continued as well.

The last important point of new research is reconsideration and correct modeling of the effect of stabilizing of the generated power in the chain of wind turbines located in the wakes one behind another. Here, attention should be paid to the impact of perturbations in the wakes of previous turbines on the development of the near wake of subsequent turbines and to the use of additional generation of various disturbances with the view of revealing the dynamics of the near wake destruction.

The above stated questions for new studies will undoubtedly give an impetus to new development of aerodynamics of wind turbines and wind farms.

The author is grateful to I.V. Naumov and V.I. Litvinov for helpful comments during the preparation of the manuscript.

\section{References}

S.V. Alekseenko, P.A. Kuibin, and V.L. Okulov, 2007, Theory of Concentrated Vortices: an Introduction, SpringerVerlag, Berlin-Heidelberg.

P. Bachant and M. Wosnik, 2015, Characterising the near-wake of a cross-flow turbine, J. Turbulence, Vol. 16, No. 4, P. 392-410.

R.J. Barthelmie, K. Hansen, S.T. Frandsen, O. Rathmann, J.G. Schepers, W. Schlez, J. Phillips, K. Rados, A. Zervos, E.S. Politis, and P.K. Chaviaropoulos, 2009, Modeling and measuring flow and wind turbine wakes in large wind farms offshore, Wind Energy, Vol. 12, No. 5, P. 431-444.

J. Bartl, F. Pierella, and L. Sætran, 2012, Wake measurements behind an array of two model wind turbines, Energy Procedia, Vol. 24, P. 305-312.

M. Bastankhan and F. Porté-Agel, 2014, A new analytical model for wind turbine wakes, Renewable Energy, Vol. 70, P. 116-123. 
M. Bastankhan and F. Porté-Agel, 2016, Experimental and theoretical study of wind turbine wakes in yawed conditions, J. Fluid Mech., Vol. 806, P. 506-541.

A. Betz, 1919, Schraubenpropeller mit geringstem Energieverlust: mit einem Zusatz von L. Prandtl, Göttinger Nachrichten 196, bis 217, Göttingen.

L.P. Chamorro, C. Hill, S. Morton, C. Ellis, R.E.A. Arndt, and F. Sotiropoulos, 2013, On the interaction between a turbulent open channel flow and an axial-flow turbine, J. Fluid Mech., Vol. 716, P. 658-670.

G.V. Ermolenko, I.G. Gordeev, A.V. Nikomarova, M.A. Ryzhenkov, and V.N. Tskhomariya, 2012, Pilot projects of network wind power in the Eisk region of Krasnodar krai: The state and prospects, Thermal, Engng, Vol. 59, No. 11, P. 846-853.

N.P. Dufresne and M. Wosnik, 2013, Velocity deficit and swirl in the turbulent wake of a wind turbine, J. Marine Technology Society, Vol. 47, No. 4, P. 193-205.

M. Felli, R. Camussi, and F. Di Felice, 2011, Mechanisms of evolution of the propeller wake in the transition and far fields, J. Fluid Mech., Vol. 682, P. 5-53.

O.A.H. Flamm, Die Schiffschraube und ihre Wirkung auf das Wasser, Berlin, 1909.

V.E. Fortov and O.S. Popel', 2014, The current status of the development of renewable energy sources worldwide and in Russia, Thermal, Engng, Vol. 61, No. 6, P. 389-398.

P. Fraunie, C. Beguier, I. Paraschivoiu, and G. Brochier, Water channel experiments of dynamic stall on Darrieus wind turbine blades, J. Propulsion and Power, 1986, Vol. 2, No. 5, P. 445-449.

R.E. Froude, 1889, On the part played in propulsion by differences of fluid pressure, Trans. Inst. Naval Architects, Vol. 30, P. 390-405

R.E. Froude, 1911, The acceleration in front of a propeller, Trans. Inst. of Naval Architects, Vol. 53, P. 139-182

Y. Fukumoto, V.L. Okulov, and D.H. Wood, 2015, The contribution of Kawada to the analytical solution for the velocity induced by a helical vortex filament, ASME Appl. Mech. Rev., Vol. 67, No. 6, P. 060801.

W.K. George, 1989, The self-preservation of turbulent flows and its relation to initial conditions and coherent structures, Advances in Turbulence, W.K. George and R. Anndt. (Eds.), Hemisphere Publ. Corp., N.Y., P. 39-73.

H. Glauert, 1935, Airplane propellers: Division L, Aerodynamic Theory IV, W.F. Durand (Ed.), P. 169-360, Springer, Berlin.

S. Goldstein, 1929, On the vortex theory of screw propellers, Proc. Roy. Soc. London A, Vol. 123, P. 440-465

A.K. Gupta, 2015, Efficient wind energy conversion: evolution to modern design, J. Energy Resour. Technol., Vol. 137, No. 5, P. 051201-1-051201-10

N. Jensen, 1983, A note on wind turbine interaction, Technical report Ris-M-2411, Roskilde, Denmark: Risø National Laboratory.

F. Jiang, H.I. Andersson, J.P. Gallardo, and V.L. Okulov, 2016, On the peculiar structure of a helical wake vortex behind an inclined prolate spheroid, J. Fluid Mech., Vol. 801, P. 1-12.

P.B. Johansson, and W.K. George, The far downstream evolution of the high-Reynolds number axisymmetric wake behind a disk. Part 1. Single-point statistics, J. Fluid Mech., 2006, Vol. 555, P. 363-385.

N.E. Joukowsky, 1912, Vortex theory of screw propeller I, Trudy Otdeleniya Fizicheskikh Nauk Obshchestva Lubitelei Estestvoznaniya, Vol. 16, Iss. 14, P. 1-31.

N.E. Joukowsky, 1914, Vortex theory of screw propeller II, Trudy Otdeleniya Fizicheskikh Nauk Obshchestva Lubitelei Estestvoznaniya, Vol. 17, Iss. 1.

N.E. Joukowsky, 1915, Vortex theory of screw propeller III, Trudy Otdeleniya Fizicheskikh Nauk Obshchestva Lubitelei Estestvoznaniya, Vol. 18, Iss. 2.

N.E. Joukowsky, 1918, Vortex theory of screw propeller IV, Trudy Avia Raschetno-Ispytatelnogo Byuro, Vol. 3-4, P. $1-97$.

M.O.L. Hansen, 2008, Aerodynamics of Wind Turbines, Earthscan (Ed.).

M. Hand, D. Simms, L. Fingersh, D. Jager, J. Cotrell, S. Schreck, and S. Larwood, 2001, Unsteady aerodynamics experiment phase vi: Wind tunnel test configurations and available data campaigns, Technical report NREL/TP. No. 500-29955.

Th. van Holten, 1981, Concentrator systems for wind energy, with emphasis on tip-vanes, Wind Engng, Vol. 5, No. 1, P. $29-45$.

J. Hong, M. Toloui, L.P. Chamorro, and M. Guala, 2014, Natural snowfall reveals large-scale flow structures in the wake of a 2.5-MW wind turbine, Nature Communications, Vol. 5, No. 4216, P. 1-9.

G.A.M. van Kuik, Momentum theory of Joukowsky actuator discs with swirl, J. Physics: Conference Series, IOP Publishing, 2016, Vol. 753, No. 2, P. 022021.

G.A.M. van Kuik, J.N. Sørensen, and V.L. Okulov, 2015, Rotor theories by professor Joukowsky: Momentum theories, Progress in Aerospace Sciences, Vol. 73, P. 1-18.

G.C. Larsen, H.A. Madsen, F. Bingöl, J. Mann, S. Ott et al., 2007, Dynamic wake meandering modeling, Risø National Laboratory.

T. J. Larsen, H.A. Madsen, G.C. Larsen, and K.S. Hansen, 2013, Validation of the dynamic wake meander model for loads and power production in the Egmond aan Zee wind farm, Wind Energy, Vol. 16, No. 4, P. 605-624.

I.V. Litvinov, I.V. Naumov, V.L. Okulov, and R.F. Mikkelsen, 2015, Comparison of far wake behind solid disk and three blades rotor, J. Flow Visualization and Image Processing, Vol. 22, No. 4, P. 175-183. 
D. Medici and P.H. Alfredsson, 2006, Measurements on a wind turbine wake: 3D effects and bluff body vortex shedding, Wind Energy, Vol. 9, P. 219-236.

I.V. Naumov, I.K. Kabardin, R.F. Mikkelsen, V.L. Okulov, and J.N. Sørensen, Performance and wake conditions of a rotor located in the wake of an obstacle, J. Phys.: Conference Series. IOP Publishing, 2016, Vol. 753, No. 3, P. 032051 .

I.V. Naumov, I.K. Kabardin, R.F. Mikkelsen, V.L. Okulov, and J.N. Sørensen, 2017, An influence of the differen incoming wake-like flows on the rotor vibrations, J. Phys.: Conference Series. IOP Publishing, Vol. 854, P. 012034

I.V. Naumov, I.V. Litvinov, R.F. Mikkelsen, and V.L. Okulov, 2015, Investigation of a wake decay behind a circular disk in a hydro channel at high Reynolds numbers, Thermophysics and Aeromechanics, Vol. 22, No.6, P. 657-665.

I.V. Naumov, I.V. Litvinov, R.F. Mikkelsen, and V.L. Okulov, 2016, Experimental investigation of wake evolution behind a couple of flat discs in a hydrochannel, Thermophysics and Aeromechanics, Vol. 23, No. 5, P. 657-666.

I.V. Naumov, R.F. Mikkelsen, and V.L. Okulov, 2016, Estimation of wake propagation behind the rotors of windpowered generators, Thermal Engineering, Vol. 63, No. 3, P. 208-213.

I.V. Naumov, R.F. Mikkelsen, V.L. Okulov, and J.N. Sørensen, 2014, PIV and LDA measurements of the wake behind a wind turbine model, J. Phys.: Conference Series, Vol. 524, P. 012168

I.V. Naumov, V.V. Rakhmanov, V.L. Okulov, K.M. Velta, K.E. Mayer, and R.F. Mikkelsen, 2012, Flow diagnostics downstream of a tribladed rotor model, Thermophysics and Aeromechanics, Vol. 19, No. 2, P. 268-278.

V.S. Neary, B. Gunawan, C. Hill, and L.P. Chamorro, 2013, Near and far field flow disturbances induced by model hydrokinetic turbine: ADV and ADP comparison, Renewable Energy, Vol. 60, P. 1-6.

B.G. Novikov, 2009, Effect of small total pulse on development of a wake behind the self-propelled bodies, Thermophysics and Aeromechanics, Vol. 16, No. 4, P. 561-583.

N.G. Nygaard, Wakes in very large wind farms and the effect of neighbouring wind farms, J. Phys.: Conference Series. IOP Publishing, 2014, Vol. 524, No. 1, P. 012162.

V.L. Okulov, Limit cases for rotor theories with Betz optimization, J. Phys.: Conference Series. IOP Publishing, 2014, Vol. 524, No. 1, P. 012129.

V.L. Okulov, 2016, An acentric rotation of two helical vortices of the same circulations, Regular and Chaotic Dynamics, Vol. 21, No. 3, P. 267-273.

V.L. Okulov, I.V. Litvinov, R.F. Mikkelsen, I.V. Naumov, and J.N. Sørensen, 2017a, Wake developments behind different configurations of passive disks and active rotors, J. Phys.: Conference Series. IOP Publishing, Vol. 854, P. 012035 .

V.L. Okulov, I.V. Litvinov, I.V. Naumov, and R. Mikkelsen, 2017b, Self-similarity of far wake behind tandem of two disks, J. Engng Thermophysics, Vol. 26, No. 2, P. 154-159.

V.L. Okulov, R.F. Mikkelsen, I.V. Litvinov, and I.V.Naumov, Efficiency of operation of wind generator rotors optimized by the Glauert and Betz methods, Techn. Phys., 2015, Vol. 60, No. 11, P. 1632-1636.

V.L. Okulov, R.F. Mikkelsen, I.V. Naumov, I.V. Litvinov, E. Gesheva, and J.N. Sørensen, 2016, Comparison of the far wake behind dual rotor and dual disk configurations, J. Phys.: Conference Series. IOP Publishing, Vol. 753, No. 3, P. 032060

V.L. Okulov, R.F. Mikkelsen, J.N. Sørensen, I.V. Naumov, and M.A. Tsoy, 2017, Power properties of two interacting wind turbine rotors, ASME. J. Energy Resour. Technol., Vol. 139, No. 5, P. 051210-1-051210-6.

V.L. Okulov, I.V. Naumov, R.F. Mikkelsen, I.K. Kabardin, and J.N. Sørensen, 2014, A regular Strouhal number for large-scale instability in the far wake of a rotor, J. Fluid Mech., Vol. 747, P. 369-380.

V.L. Okulov, I.V. Naumov, R.F. Mikkelsen, and J.N. Sørensen, 2015, Wake effect on a uniform flow behind windturbine model, J. Phys.: Conference Series. IOP Publishing, Vol. 625, No. 1, P. 012011.

V.L. Okulov, I.V. Naumov, and J.N. Sørensen, 2007, Optical diagnostics of intermittent flows, Techn. Phys., Vol. 77, No. 5, P. 47-57.

V.L. Okulov, I.V. Naumov, M.A. Tsoi, and R.F. Mikkelsen, 2017, Loss of efficiency in coaxial arrangement of the pair of wind turbines, Thermophysics and Aeromechanics, Vol. 24, No. 4, P. 545-551.

V.L. Okulov and J.N. Sorensen, 2007, Stability of helical tip vortices in a rotor far wake, J. Fluid Mech., Vol. 576 , P. 1-25.

V.L. Okulov and J.N. Sorensen, 2008, Refined betz limit for rotors with a finite number of blades, Wind Energy, Vol. 11, No. 4, P. 415-426.

V.L. Okulov and J.N. Sorensen, 2010a, Applications of 2D helical vortex dynamics, Theor. Comput. Fluid Dyn., Vol. 24, P. 395-401.

V.L. Okulov and J.N. Sorensen, 2010b, Maximum efficiency of wind turbine rotors using Joukowsky and Betz approaches, J. Fluid Mech., Vol. 649, P. 497-508.

V.L. Okulov, J.N. Sorensen, and W.Z. Shen, 2016, Expansion of Goldstein's circulation function for optimal rotors with hub, J. Phys.: Conference Series. IOP Publishing, Vol. 753, No. 2, P. 022018.

V.L. Okulov, J.N. Sørensen, and G.A.M. van Kuik, 2013, Development of the Optimum Rotor Theories, MoscowIzhevsk: R\&C Dyn.

V.L. Okulov, J.N. Sorensen, and D.H. Wood, 2015, The rotor theories by professor Joukowsky: Vortex theories, Progress in Aerospace Sci., Vol. 73, P. 19-46. 
H.U. Quaranta and T. Leweke, 2015, Long-wave instability of a helical vortex, J. Fluid Mech., Vol. 780, P. 687-716.

W.J.M. Rankine, 1865, On the mechanical principles of the action of propellers, Trans. Inst. Naval Architects, Vol. 6 , P. 13-39.

A.Segalini and P. Inghels, 2014, Confinement effects in wind-turbine and propeller measurements, J. Fluid Mech. Vol. 756, P. 110-129.

M.S. Selig, J.J. Guglielmo, A.P. Broeren, and P. Giguere, 1995, Summary of Low-Speed Airfoil Data, Vol. 1, SolarTech Publication.

J.N. Sørensen, 2016, General Momentum Theory for Horizontal Axis Wind Turbines, Springer.

J.N. Sørensen and G.A.M. van Kuik, 2010, General momentum theory for wind turbines at low tip speed ratios, Wind Energy, Vol. 14, P. 821-839.

J.N. Sørensen, R.F. Mikkelsen, N. Troldborg, V. Okulov, and W.Z. Shen, 2013, The aerodynamics of wind turbines, in: Proc. 22nd Int. Congress of Theoretical and Applied Mechanics, ICTAM 2008, P. 231-247, Mechanics Down Under.

J.N. Sørensen, V.L. Okulov, R.F. Mikkelsen, I.V. Naumov, and I.V. Litvinov, 2016, Comparison of classical methods for blade design and the influence of tip correction on rotor performance, J. Phys.: Conference Series. IOP Publishing, Vol. 753, No. 2, P. 022020.

B.M. Sumer and J. Fredøse, 2006, Hydrodynamics around cylindrical structures, Advanced Series on Ocean Engineering 26, World Scientific.

B. Sunden and Z. Wu, 2015, On icing and icing mitigation of wind turbine blades in cold climate, J. Energy Resour. Technol., Vol. 137, No. 5, P. 051203-1-051203-10.

K.W. van Treuren, 2015, Small-scale wind turbine testing in wind tunnels under low Reynolds number conditions, J. Energy Resour. Technol., Vol. 137, No. 5, P. 051208-1-051208-11.

L. Vermeer, J.N. Sørensen, and A. Crespo, 2003, Wind turbine wake aerodynamics, Progress in Aerospace Sci., Vol. 39, P. 467-510.

O.F. Voropaeva, O.A. Druzhinin, and G.G. Chernykh, 2016, Numerical simulation of momentumless turbulent wake dynamics in linearly stratified medium, J. Engng Thermophysics, Vol. 25, No. 1, P. 85-99.

Q.R. Wald, 2006, The aerodynamics of propellers, Progress in Aerospace Sci., Vol. 42, No. 2, P. 85-128.

J.H. Walther, M. Guenot, E. Machefaux, J.T. Rasmucsen et al., 2007, A numerical study of the stabilitiy of helical vortices using vortex methods, J. Phys.: Conference Series. IOP Publishing, Vol. 75, No. 1, P. 012034.

D.H. Wood and V.L. Okulov, 2017, Nonlinear blade element-momentum analysis of Betz-Goldstein rotors, Renewable Energy, Vol. 107, P. 542-549.

D.H. Wood, V.L. Okulov, and D. Bhattacharjee, 2016, Direct calculation of wind turbine tip loss, Renewable Energy, Vol. 95, P. 269-276.

J. Yang, M. Liu, G. Wu, W. Zhong, and X. Zhang, 2014, Numerical study on coherent structure behind a circular disk, J. Fluids Struct., Vol. 51, P. 172-188. 